\title{
The Energetic Cost of Adaptive Feet in Walking
}

\author{
Seungmoon Song and Hartmut Geyer
}

\begin{abstract}
While humanoid feet are made of rigid plates, human feet have evolved into highly articulated and flexible elements. This adaptiveness provides key advantages. It absorbs impacts and secures grip when interacting with the environment. However, the human foot design potentially increases the energetic cost, because it features actuators and provides less power transfer than a rigid plate does. Here we use neuromuscular models with different foot designs and show that human feet incur about $20 \%$ more energetic cost than rigid ones for walking speeds up to $1.2 \mathrm{~ms}^{-1}$, which is close to the preferred walking speed. Above this speed, human feet do not show an energetic disadvantage. In addition we propose a foot design for prosthetic or humanoid feet which preserves key features of adaptive feet but does not require actuation, and show that it reduces the energetic cost by $15 \%$ or more independent of the walking speed. We conclude that human evolution may have traded the advantages of adaptive feet for energy efficiency, and that robotic systems could gain the former without compromising on the latter.
\end{abstract}

\section{INTRODUCTION}

The foot plays a special role in human locomotion. It is the segment that interacts with the environment, taking impacts, securing grip, and transmitting power. Along with this special role, the human foot has evolved, as already Leonardo da Vinci observed, into a "masterpiece of engineering" [1] that comprises about 30 segments and joints, 20 musclestendon units, and over 100 ligaments [2]. In comparison to this rich biomechanical design, the feet of humanoid robots seem grossly oversimplified. They mainly consists of single segments with added rubber pads that feature neither internal joints and segmentation nor internal actuation [3]-[8], hinting at an ample potential for improving the stability, agility and performance of humanoid locomotion.

The potential has been recognized and the biomechanics of the human foot attracts a growing interest in robotics research. For example, [9] have recently demonstrated in experiments with the WABIAN humanoid that a human-like tripod design of the foot contact area improves static stability over a traditional humanoid rectangular foot in uneven terrain where three-point contacts are common, because the tripod design keeps the support area around the center of the foot. Furthermore, an internal toe joint has been explored in several robotics studies to provide additional propulsion [10], [11] or to alter the foot segment stiffness in different phases of stance [12].

The last example is motivated by biomechanical studies that have revealed less obvious foot designs expressing important functions. Because human feet contain many internal

S. Song and H. Geyer are with the Robotics Institute, Carnegie Mellon University, 5000 Forbes Avenue, Pittsburgh, PA 15213, USA. \{smsong, hgeyer\} @ cmu.edu joints, they are more flexible than other leg segments. This flexibility allows to cushion impact and secure foothold, but it is detrimental to effective power transfer. Experimental studies suggest that this problem is partially solved in human feet by the windlass mechanism [13], which passively stiffens the longitudinal foot arch [14], and the cushioning soft tissue below the foot, when the foot rolls over the toe during push-off [15]. In addition to the windlass mechanism, the toe adds more functional advantages. It allows to alter the foot length, combining effective center of pressure control in stance [16] with ground clearance in swing, and to improve grip by converting horizontal endpoint forces into vertical ones when the ankle pushes off.

The added functionality of the foot does not come for free, however. The windlass mechanism does not create a perfectly rigid foot element at push-off. Nor does the toe flex and extend on its own, requiring additional actuation. Thus the added functionality likely compromises energy efficiency. But how much should humanoids compromise on energy efficiency for the added functionality?

Here we try to answer this question by investigating how much humans are willing to compromise. We present two neuromuscular walking models that feature different foot designs and use optimization to compute their minimum energetic cost at speeds ranging from slow walking at $0.8 \mathrm{~ms}^{-1}$ to fast walking at $1.8 \mathrm{~ms}^{-1}$ (section II \& III). The two designs are a rigid arched foot that provides a baseline measure for energy expenditure, and a human-like foot that can flex the foot arch, and adds the windlass mechanism as well as an actuated toe. In addition, we present a third model whose foot design implements two key features of articulated adaptive feet, the windlass mechanism in stance and the toe lift in swing, with a passive design that can be used in humanoids and prosthetic feet without spending additional energy on toe actuators. Our results indicate that although human feet have no detrimental effect on energy efficiency above a speed of $1.2 \mathrm{~ms}^{-1}$, up to this normal walking speed they incur about $20 \%$ more energetic cost than rigid feet (section IV). By contrast we find that the third foot design reduces the cost by $15 \%$ or more independent of the walking speed. These results suggest that humans are willing to pay a substantial price in energetic cost for adaptive feet and we discuss how the third, passive design could help to alleviate some of that cost in robotic implementations (section V).

\section{Walking Models with Alternative Feet}

In previous work, we have identified a forward-dynamic human model of neuromuscular control that generates steady-state walking behavior with human-like kinematics, 
kinetics, and muscle activations [17] (Fig. 1). This model represents the human musculoskeletal system as a planar, seven segment system modeling the trunk as well as the thighs, shanks and feet. The segments are connected by revolute joints forming the hip, knee and ankle. The joints are actuated by seven Hill-type muscle models per leg, representing major leg muscles in human walking (soleus, SOL; gastrocnemius, GAS; tibialis anterior, TA; vastii group, VAS; hamstring group, HAM; gluteus maximus, GLU; and grouped hip flexors, HFL; Fig. 1). The muscle models consist of contractile elements, who take muscle stimulation signals $S_{m}$ from $0 \%$ to $100 \%$ as input, combined with series and parallel elasticities. Each muscle's force translates into a joint torque contribution $\tau_{m, j}=F_{m} r_{m}\left(\varphi_{j}\right)$ at the joints $j$ it spans, using variable moment arms $r_{m}\left(\varphi_{j}\right)$ that mimic the physiological moment arms observed for these muscles and joints.

The muscle stimulations $S_{m}$ are generated by the model's neural feedback control. The control consists of separate stance and swing phase reflexes which are based on sensory signals measuring the muscle state (mostly homonymous, positive force or length feedbacks, $F+$ or $L+$, Fig. 1). To reflect neural transport delays, these signals are time-delayed, as well as gained, and fed back into sum blocks that model alpha motoneurons and produce the muscle stimulations. The sensory feedback pathways that are used in the model have been synthesized element by element from translating a bipedal spring-mass model [18] into an articulated one, and encoding compliant leg behavior and other principles of legged dynamics and control into muscle reflexes control (see [17] for details on this model).

While in the previous version of this model the foot is a flat rigid segment, we here replace it by three alternative designs to investigate the effect of adaptive feet on the energetic efficiency. The first design is a rigid arched foot (Fig. 2a) that serves as a baseline model when computing the energetic cost of locomotion. The second and third designs implement a foot that can flex the arch and adds the windlass mechanism (Fig. 2b). The flexible foot arch is realized by a spring loaded midtarsal joint (MTJ, $k_{M T J}=0.8 k N \mathrm{mrad}^{-1}$ ). The windlass mechanism combines the flexible arch with the plantar fascia $\left(\mathrm{PF}, k_{P F}=5 \cdot 1000 \mathrm{kNm}^{-2}, l_{\text {slack }}=16 \mathrm{~cm}\right)$, a tendon spanning from the heel around the metatarsalphalangeal joint (MTPJ) to the toe segment [13], [19]-[21]. When the foot rolls over the toe at push-off, the PF tensions and pulls the ball about the MTJ toward the heel, stiffening the foot arch for effective power transfer [22], [23]. On the other hand, the PF also tensions when the body weight loads the foot in stance and the ball and heel get pushed apart, passively exerting toe flexion torque that creates center of pressure control. Note that PF stretch is substantial in human walking where it reaches one to two centimeters [13], [19]. All three foot designs share the same overall length (unloaded PF for the adaptive feet) and moment of inertia with respect to the ankle (Tab. I).

The second and third designs differ in the way toe actuation is achieved. It is implemented in the second, human-

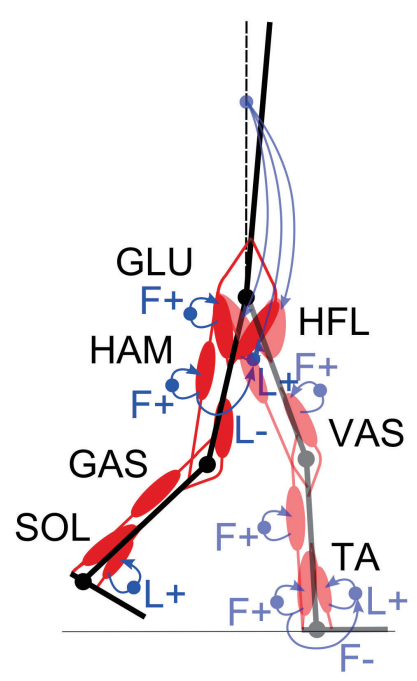

Fig. 1. Neuromuscular human walking model. The model has seven segments driven by 14 muscles. Segment masses and inertias reflect human data. Muscles are modeled as Hill-type muscles with force-length and force-velocity relationships, and include series and parallel springs. The control is purely reflexive. It does not require central pattern generators or precomputed joint trajectories. Key muscle reflexes active in swing (shown for left leg) are the positive length feedback (L+) of tibialis anterior (TA) lifting the foot, $\mathrm{L}+$ of the hip flexor and its suppression by positive force feedback $(F+)$ of the biarticular hamstring (HAM) and the glutei (GLU). Key reflexes in stance (right leg) are $F+$ of the leg extensors soleus (SOL), gastrocmenius (GAS) and vastii (VAS), negative force feedback $(F-)$ from SOL to TA suppressing the TA L+, and the trunk balance control around a reference lean from the vertical that activates either GLU and HAM or HFL based on a proportional-derivative signal of the forward lean.

TABLE I

MECHANICAL PARAMETERS FOR THE THREE DIFFERENT FOOT DESIGNS (BASELINE, HUMAN AND PASSIVE).

\begin{tabular}{l|c|ccc} 
& Baseline & \multicolumn{3}{|c}{ Human \& Passive } \\
& foot & rearfoot & midfoot & toe \\
\hline$l_{S x}, l_{S y}(\mathrm{~cm})$ & 25,0 & 4,8 & 16,8 & 5,0 \\
$d_{G, S x}, d_{G, S y}(\mathrm{~cm})$ & 19,7 & 3,6 & 15,7 & 3,0 \\
$m_{S}(\mathrm{~kg})$ & 1.25 & 0.35 & 0.8 & 0.1 \\
$\Theta_{S}\left(\mathrm{kgm}^{2}\right)$ & 0.0045 & 0.0001 & 0.0007 & 0.0001
\end{tabular}

like, design by two muscles that originate from the shank (Fig. 2c). Although human feet are actuated by intrinsic foot muscles as well, we neglect these smaller muscles and focus on the large multiarticular toe flexor group (TFL, $\left.\left\{F_{\max }, v_{\max }, l_{\text {opt }}, l_{\text {slack }}\right\}=\left\{900 \mathrm{~N}, 12 l_{\text {opt }} \mathrm{s}^{-1}, 4 \mathrm{~cm}, 9 \mathrm{~cm}\right\}\right)$ and extensor muscle (TEX, $\left\{F_{\max }, v_{\max }, l_{\text {opt }}, l_{\text {slack }}\right\}=$ $\left.\left\{500 \mathrm{~N}, 12 l_{\text {opt }} s^{-1}, 8 \mathrm{~cm}, 22 \mathrm{~cm}\right\}\right)$. TFL and TEX originate from the shank and span via long tendons the ankle, MTJ and MTPJ, before inserting in the toe (see Tab. II for muscle-skeleton link parameters). The control of these two actuators mirrors the control identified for SOL and TA in the previous model [17]. During stance, the TFL is controlled by homonymous $F+$, automatically reinforcing actuator output with increasing ground interaction forces at the toe, $S_{T F L}=S_{0, T F L}+G_{T F L} F_{T F L}\left(t_{l}\right)$, where $S_{0, T F L}$ is the prestimulation, $G_{T F L}$ is the force feedback gain, and 


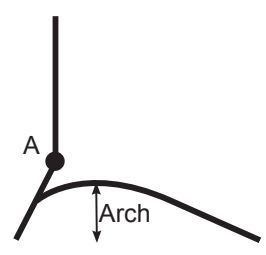

(a) Baseline

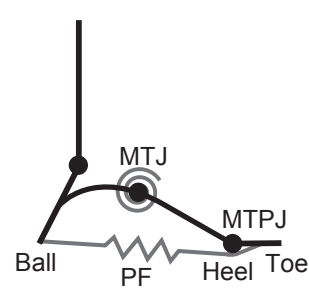

(b)

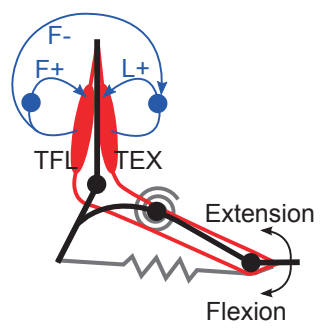

(c) Human

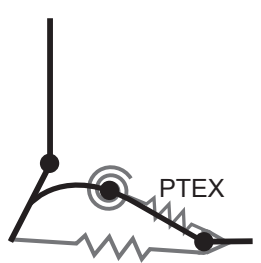

(d) Passive

Fig. 2. Alternative foot designs. (a) Rigid arched foot used as baseline model for energetic cost comparisons. (b) Windlass mechanism. (c) Human-like adaptive foot that includes the windlass mechanism and toe actuation. (d) Passive adaptive foot that preserves the windlass mechanism and adds a passive toe extension spring for automatic toe lift in swing.

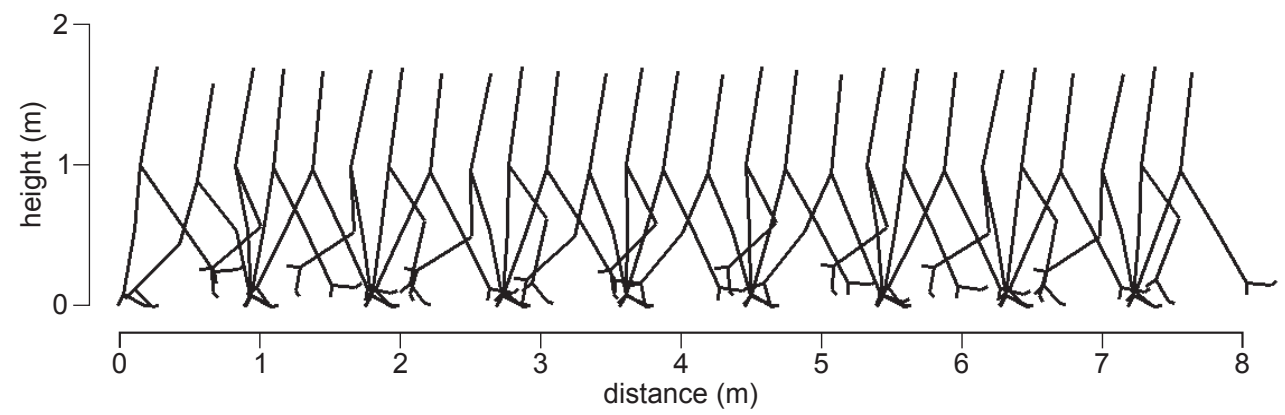

Fig. 3. Neuromuscular model walking with human foot design. Snap shots of the model are shown for every $200 \mathrm{~ms}$ of simulation. The model starts with $1 \%$ muscle activation for all its muscles at initial values of the ankle, knee, and hip angles equal to $85^{\circ}, 175^{\circ}$ and $175^{\circ}$ for left leg, and $90^{\circ}, 175^{\circ}$ and $140^{\circ}$ for right leg). The control stabilizes the model into steady walking at about $1.3 \mathrm{~ms}^{-1}$ within a few steps.

$F_{T F L}\left(t_{l}\right)$ is the delayed signal of the force exerted by TFL for $t_{l}=t-20 \mathrm{~ms}$. During swing, the TFL is inactive. By contrast, TEX is controlled throughout the gait cycle using homonymous $\mathrm{L}+$, which tends to lift the foot with the toe into a reference position, providing ground clearance. This feedback control is inhibited during stance via a negative force feedback $F$ - from the TFL to avoid counteracting its action, $S_{T E X}=S_{0, T E X}+G_{T E X}\left[l_{C E, T E X}\left(t_{l}\right)-\right.$ $\left.l_{\text {off,TEX}}\right]-G_{T F L T E X} F_{T F L}\left(t_{l}\right)$, where $G_{T E X}$ is the length feedback gain, $l_{C E, T E X}\left(t_{l}\right)$ is the delayed signal of the TFL fiber length, $l_{\text {off,TEX }}$ is the length offset, and $G_{T F L T E X} F_{T F L}\left(t_{l}\right)$ is the inhibition term proportional to TFL force (which is zero during swing). Note that the feedback controls for TFL and TEX include a time delay of $20 \mathrm{~ms}$ reflecting the transport delays of neural signals in human motor control.

The third design, in contrast, does not have muscle actuation (Fig. 2d). It relies instead on passive springs, the PF and a passive toe extensor spring (PTEX, $k_{P T E X}=100 \mathrm{kNm}^{-2}$, $l_{\text {slack }}=5 \mathrm{~cm}$ ), to actuate the toe. The torque balance between PTEX and PF is tuned so that PTEX lifts the toe in swing into about 20 degree toe extension.

We implement the three model extensions as different neuromuscular models in the MatLab Simulink/SimMechanics environment (R2010a) using the ode15s solver and custom nonlinear contact models [17] at the heel, ball (only human and passive foot design) and toe of each foot that simulate ground contact dynamics. Fig. 3 shows as a characteristic
TABLE II

MUSCLE-SKELETON ATTACHMENT PARAMETERS INCLUDING MOMENT ARM $r_{0}$, MAXIMUM AND REFERENCE ANGLE, $\varphi_{\max }$ AND $\varphi_{r e f}$, AND SCALING FACTOR $\rho$ AS USED IN [17].

\begin{tabular}{l|ll|llll|lll|l} 
& \multicolumn{1}{|c}{$\begin{array}{c}\text { Human } \\
\text { ankle }\end{array}$} & \multicolumn{4}{c|}{$\begin{array}{c}\text { MTJ } \\
\text { MTPJ }\end{array}$} & $\begin{array}{l}\text { Passive } \\
\text { MTPJ }\end{array}$ \\
& TEXTFL & TA & TEXTFL PF & TEXTFL PF & PTEX \\
\hline$r_{0}(\mathrm{~cm})$ & 4 & 2 & 1 & 1 & 6 & 6 & 1 & 1 & 1 & 1 \\
$\varphi_{\max }(\mathrm{deg})$ & 80 & 110 & - & - & - & - & - & - & - & - \\
$\varphi_{\text {ref }}(\mathrm{deg})$ & 90 & 90 & 0 & 0 & 0 & 0 & 20 & -10 & 0 & 50 \\
$\rho$ & 0.7 & 0.7 & 0.7 & 0.7 & 0.7 & 0.7 & 0.7 & 0.7 & 1 & 0.7
\end{tabular}

example of the resulting motion the neuromuscular model with the human-like foot walking at about $1.3 \mathrm{~ms}^{-1}$.

\section{Energetic Cost Optimization}

To compare the effect of the different ankle-foot configurations on energy consumption in walking, we use CMAES optimization [24] of the models' control parameters. We optimize all 30 control parameters ( +6 for the human toe muscle control) that can be classified into stance leg, swing leg, and trunk balance parameters (Tab. III). In each generation, our implementation of the CMA-ES samples a population of 16 solutions from this space of control parameters and initial conditions according to a multivariate normal distribution, evaluates these solutions based on a cost function, and uses the 8 best solutions to reshape the covariance matrix of the normal distribution. This procedure repeats for 400 generations. 
TABLE III

CONTROL PARAMETERS OF THE NEUROMUSCULAR MODEL USED IN THE OPTIMIZATION, CLASSIFIED BY STANCE LEG, SWING LEG, AND TRUNK BALANCE CONTROL. IN ADDITION, THE PRESTIMULATION OF ALL MUSCLES ARE INCLUDED AS CONTROL PARAMETERS.

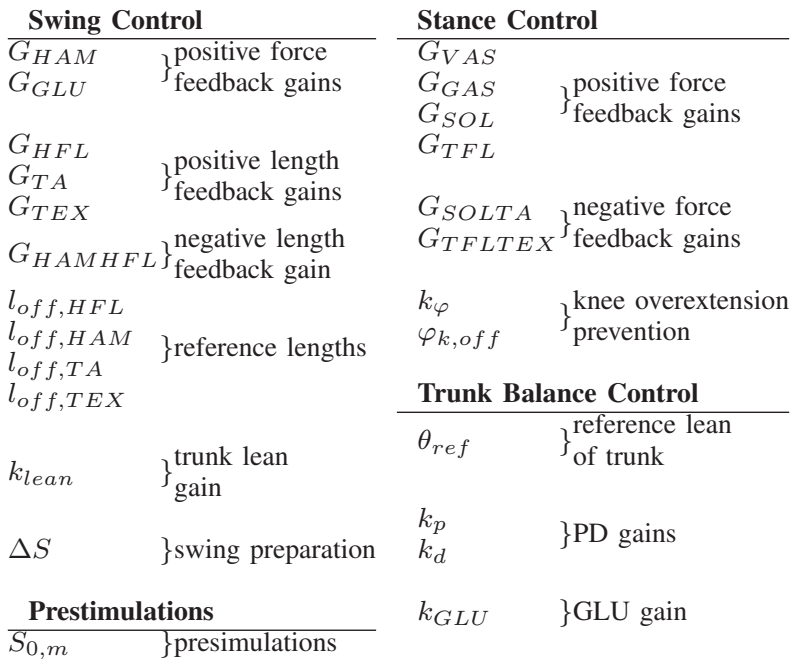

As cost function we use

$$
J=10\left|\dot{x}_{a v g}-\dot{x}_{t g t}\right|+P+C_{E}
$$

where $\dot{x}_{t g t}$ and $\dot{x}_{a v g}$ are a target walking speed and the average walking speed, $P$ is a pain term that accounts for joint over-extensions, and $C_{E}$ is the energetic cost. We estimate $P$ as the sum of torques created by the soft mechanical joint limits in the neuromuscular model, which engage outside of the normal joint ranges $\left(70^{\circ}<\varphi_{a}<130^{\circ}\right.$, $\varphi_{k}<175^{\circ}$, and $\varphi_{h}<230^{\circ}$ for the ankle, knee and hip, respectively [17]). We compute the energetic cost as $C_{E}=E_{M} /\left(m x_{d}\right)$, where $m$ is the body mass, $x_{d}$ is the distance travelled, and $E_{M}$ is the total metabolic energy. $E_{M}$ is estimated as the sum of metabolic energies expended by all muscles, using the muscle energy model of [25]. We assume that an individual simulation has achieved steady walking as soon as the leg joint positions do not change significantly relative to the touch-down position between subsequent heelstrikes (sum of differences $\leq 3 \mathrm{~cm}$ proved a reliable indicator of steady walking in extensive test simulations; note that if no steady state is achieved, the simulation is replaced by one of an additional sample of the search space). For the three subsequent steady-state gait cycles, we compute the average speed, pain and energetic cost to determine the cost function $J$ (Eq. 1).

We optimize all three models for six walking speeds ranging from slow walking at $0.8 \mathrm{~ms}^{-1}$ to fast walking at $1.8 \mathrm{~ms}^{-1}$. Each optimization trial starts after the model simulation achieves steady walking when initialized with a known control parameter set. For the first optimization at $1.4 \mathrm{~ms}^{-1}$, we use hand-tuned parameters for this known set; in subsequent optimizations, we recursively branch out to target speeds that are $0.2 \mathrm{~ms}^{-1}$ above and below, and

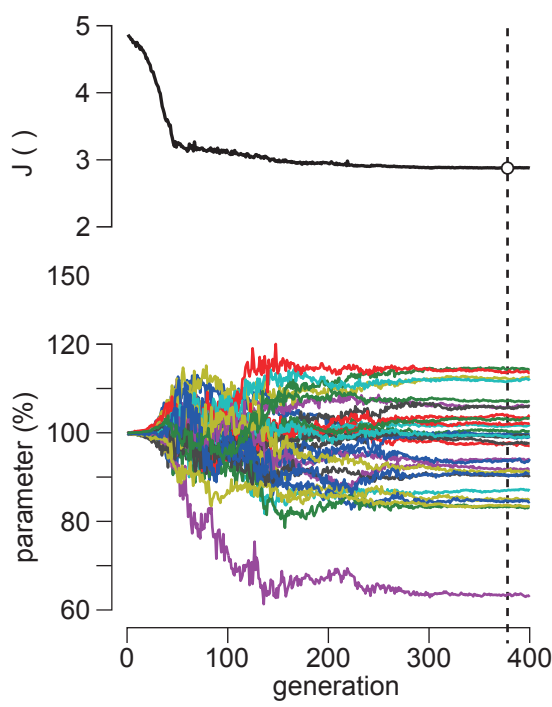

Fig. 4. Characteristic example of the cost and parameter evolution during optimization. The example shows the first optimization run for the walking model with the baseline foot (Fig. 2a) for a target speed of $1.4 \mathrm{~ms}^{-1}$. The top panel shows the evolution of the lowest achieved cost $J$. The cost changes substantially during the first 100 generations and levels off after about 250 generations. The minimum cost is found after 380 generations (circle). The bottom panel shows the corresponding evolution of the control parameters, which equally settle after 250 generations.

use as initial parameter sets the ones that the optimization found at neighboring target speeds. In addition, we repeat the optimization for each target speed to verify the results. (For all speeds, we did not observe significant changes in the minimum cost between the two optimization runs.)

Figure 4 provides a characteristic example of the parameter evolution during optimization. Significant changes in the control parameters and the cost function $J$ occur in the first 200 generations. After that only minor changes occur and the cost function settles to the energetic cost.

\section{RESUlTS}

The energetic cost optimization for the three neuromuscular models with the different ankle-foot configurations reveals that human feet incur about 20\% more energetic cost than rigid ones for slow to moderate walking speeds. Figure 5 shows the identified minimum energetic cost at the six target speeds and figure 6 compares the relative cost with the baseline model as the reference. For speeds up to a typical human walking speed of $1.2 \mathrm{~ms}^{-1}$, the minimum energetic cost for the model with the human-like foot are the highest among all three models (Fig. 5). In particular, it is at an energetic disadvantage of about $20 \%$ relative to the baseline model with a rigid foot (Fig. 6). Although the costs are comparable between the two models at speeds larger than $1.2 \mathrm{~ms}^{-1}$, the significant higher cost of human feet at slow to moderate speeds indicates that human evolution may have traded the functional advantages of adaptive feet for energy efficiency.

In contrast, the model with the passive foot design that implements some key features of adaptive feet shows significantly reduced energetic cost throughout all walking speeds. 


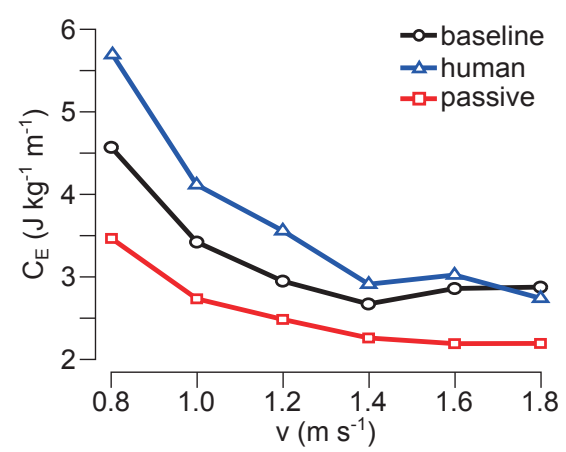

Fig. 5. Energetic cost for walking with different feet. The minimum energetic costs are shown at six target speeds for the neuromuscular walking models with the baseline, human, and passive foot configurations. For comparison, humans consume $3.3-3.6 \mathrm{Jkg}^{-1} \mathrm{~m}^{-1}$ at a normal speed of $1.2 \mathrm{~ms}^{-1}[26]$.

The model has the lowest absolute cost among all three models (Fig. 5), generating a relative cost advantage of at least $15 \%$ over the baseline model (Fig. 6) and of at least $20 \%$ over the human-like model (not explicitly shown in Fig. 6).

\section{COnClusion And Future Work}

Human-like adaptive feet provide advantages, for instance, by reducing impact and securing grip, but their need for actuation and their reduced rigidity could have a negative effect on the energy efficiency in walking. While humans seem to be willing to pay for this extra cost, humanoids do not have to and still can reap some gains that adaptive feet provide. The third foot design that we investigated takes advantage of the windlass mechanism and of the toe lift in swing without requiring active control. It could provide a viable alternative for humanoid or prosthetic feet.

In future research, we plan to expand on this work in two ways. First, we plan to develop a robotic foot that realizes the passive foot model and to test in experiments the predicted benefit on energy efficiency. Second, we will investigate in the neuromuscular models how the essential changes in the control parameters between the steady walking speeds that the optimization identified can be converted into a reflexively driven speed control. Such an autonomous adaptation to speed changes not only would increase the versatility of the neuromuscular models, but also could guide the development of controllers for powered prosthetic legs.

\section{ACKNOWLEDGMENTS}

This work is sponsored by the DARPA Maximum Mobility and Manipulation Program under contract W911NR-11-10098. The authors would like to thank M. Günther for helpful comments on foot models and N. Hansen for clarifications on CMA-ES implementations.

\section{REFERENCES}

[1] L. da Vinci. (2011, June) Great-quotes.com. [Online]. Available: http://www.great-quotes.com/quote/52031

[2] P. Channel. (2011, June) Anatomy of the foot and ankle. [Online]. Available: http://www.podiatrychannel.com

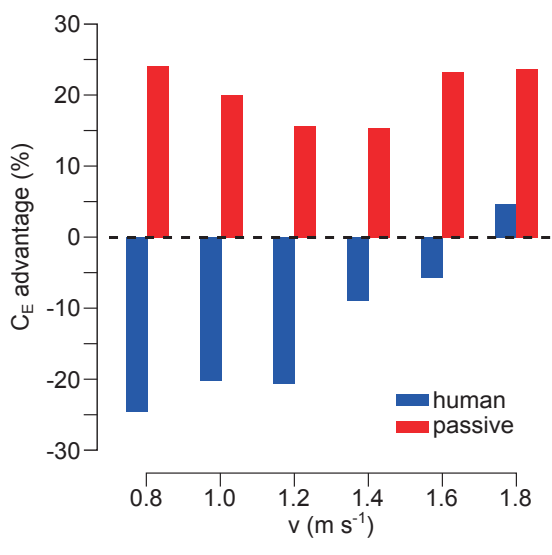

Fig. 6. Energetic cost relative to the baseline model. The model with the human-like foot incurs at least $20 \%$ more energetic cost than the baseline model for walking speeds up to $1.2 \mathrm{~ms}^{-1}$, but shows only small differences for faster walking speeds. In contrast, the passive foot model has at least $15 \%$ advantage in energetic cost over the baseline model throughout all walking speeds.

[3] Y. S. et al., "The intelligent asimo: system overview and integration," in Proc. IEEE/RSJ Int. Conf. Intel. Rob. Syst., 2002.

[4] J. K. et al., "System design and dynamic walking of humanoid robot khr-2," in Proc. IEEE Int. Conf. Rob. Automat., 2005.

[5] K. K. et al., "Humanoid robot hrp-2," in Proc. IEEE Int. Conf. Rob. Automat., 2004.

[6] D. Hobbelen, T. de Boer, and M. Wisse, "System overview of bipedal robots flame and tulip: tailor-made for limit cycle walking," in Proc. IEEE/RSJ Int. Conf. Intel. Rob. Syst., 2008.

[7] B. Stephens and C. Atkeson, "Modeling and control of periodic humanoid balance using the linear biped model," in Proc. IEEE-RAS Int. Conf. Intel. Humanoid Rob., 2009.

[8] S. Song, Y. Ryoo, and D. Hong, "Development of an omnidirectional walking engine for full-sized lightweight humanoid robots," in Proc. ASME Int. Design Eng. Tech. Conf. Comp. Inform. Eng. Conf., 2011, in press.

[9] H. K. et al., "Realization of biped walking on uneven terrain by new foot mechanism capable of detecting ground surface," in Proc. IEEE Int. Conf. Rob. Automat., 2010.

[10] W. Zhang, F. Chen, M. Li, and Q. Huang, "Simulation of humanoid motion based on the foot with one active joint," in Proc. IEEE Int. Conf. Rob. Biomim., 2010.

[11] J. Zhu, Q. Wang, Y. Huang, and L. Wang, "Adding compliant joints and segmented foot to bio-inspired below-knee exoskeleton," in Proc. IEEE Int. Conf. Rob. Automat., 2011.

[12] K. H. et al., "A study of function of foot's medial longitudinal arch using biped humanoid robot," in Proc. IEEE/RSJ Int. Conf. Intel. Rob. Syst., 2010.

[13] J. H. Hicks, "The mechanics of the foot - ii. the plantar aponeurosis and the arch," J. Anat., vol. 88, no. 1, pp. 25-30, 1954.

[14] P. J. Briggs and P. A. Tansey, "Active and passive mechanisms in the control of supination," Foot Ankle Surg., vol. 7, pp. 131-136, 2001.

[15] F. Bojsen-Moller and K. E. Flagstad, "Plantar aponeurosis and internal architecture of the ball of the foot," J. Anat., vol. 121, no. 3, pp. 599$611,1976$.

[16] D. A. Winter, "Human balance and posture control during standing and walking," Gait and Posture, vol. 3, no. 4, 1995.

[17] H. Geyer and H. M. Herr, "A muscle-reflex model that encodes principles of legged mechanics produces human walking dynamics and muscle activities," IEEE Trans. Neural Syst. Rehab. Eng., vol. 18, no. 3, 2010.

[18] H. Geyer, A. Seyfarth, and R. Blickhan, "Positive force feedback in bouncing gaits?” Proc. R. Soc. B, vol. 270, pp. 2173-2183, 2003.

[19] L. A. Bolgla and T. R. Malone, "Plantar fasciitis and the windlass mechanism: a biomechanical link to clinical practice," J. Athlet. Train. Assoc., vol. 39, no. 1, pp. 77-82, 2004.

[20] A. Erdemir, A. J. Hamel, A. R. Fauth, S. J. Piazza, and N. A. Sharkey, "Dynamic loading of the plantar aponeurosis in walking," J. Bone Joint Surg., vol. 86, no. 3, pp. 546-552, 2004. 
[21] P. Caravaggi, T. Pataky, J. Y. Goulermas, R. Savage, and R. Crompton, "A dynamic model of the windlass mechanism of the foot: evidence for early stance phase preloading of the plantar aponeurosis," J. Experim. Biol., vol. 212, pp. 2491-2499, 2009.

[22] F. Bojsen-Moller, "Calcaneocuboid joint and stability of the longitudinal arch of the foot at high and low gear push off," J. Anat., vol. 129, no. 1, pp. 165-176, 1979.

[23] J. M. Czerniecki, "Foot and ankle biomechanics in walking and running: a review," J. Anat., vol. 67, no. 6, pp. 246-252, 1988.

[24] N. Hansen, "The cma evolution strategy: A comparing review," Towards a New Evolutionary Computation. Advances on Estimation of Distribution Algorithms. Springer, p. 75.102, 2006.

[25] B. R. Umberger, K. G. Gerritsen, and P. E. Martin, "A model of human muscle energy expenditure," Comp. Meth. Biomech. Biomedic. Eng., vol. 6, no. 2, pp. 99-111, 2003.

[26] R. L. Waters and S. Mulroy, "The energy expenditure of normal and pathological gait," Gait and Posture, vol. 9, pp. 207-231, 1999. 